\title{
The experience of executing preventive measures to protect a nursing home in Taiwan from a COVID-19 outbreak
}

\author{
Chia-Yu Huang ${ }^{1,2,3} \cdot$ Yu-Hung Kuo ${ }^{4} \cdot$ Shu-Ting Chuang ${ }^{5,6} \cdot$ Hung-Rong Yen $^{3,7,8,9} \cdot$ Sio-lan Tou $^{10}$ (1)
}

Received: 27 October 2020 / Accepted: 26 January 2021 / Published online: 22 March 2021

(c) European Geriatric Medicine Society 2021

\section{Key summary points}

Aim We share our strategies for preventing a COVID-19 outbreak in a nursing home in Taiwan and evaluate the efficacy of these strategies.

Findings (1) Our strategies are useful for decreasing the numbers of outpatient department visits, days of prescription from the outpatient department, number of emergency department visits, number of admissions, and days of admission to treat respiratory tract infections in the nursing home residents. (2) Nursing home staff also had lower numbers of outpatient department visits and days of prescription from the outpatient department to treat respiratory tract infections through executing our preventive measures for a COVID-19 outbreak.

Message Our preventive strategies for the COVID-19 outbreak are helpful for decreasing the incidence and severity of respiratory tract infections in the nursing home residents and staff. Taiwan's experience could also be a benchmark for other countries. However, these strategies still require weeks to achieve their estimated results.

\begin{abstract}
Purpose We share our strategies for preventing the COVID-19 outbreak in a nursing home in Taiwan.

Methods We compared the number of outpatient department visits, the days of prescription from the outpatient department, the number of emergency department visits of the nursing home residents and staff, the number of admissions, and the days of admission of the residents for respiratory tract infection treatment between 2019 and 2020 to examine the effect of our preventive measures in the nursing home. Residents and staff who continuously lived and worked in the nursing home from 2019 to 2020 were included. The differences in outcomes between 2019 and 2020 were examined using paired sample t tests. The multivariate analyses were presented through generalized estimating equation analysis.

Results A cohort of 183 residents and 127 staff was included and their electronic medical documentation was analyzed in two periods: January-September 2019 and January-September 2020. These residents had lower numbers of outpatient department
\end{abstract}

Hung-Rong Yen and Sio-Ian Tou contributed equally to this work.

Sio-Ian Tou

dorislovenel@gmail.com

1 Department of Family Medicine, Taichung Tzu Chi Hospital, Buddhist Tzu Chi Medical Foundation, Taichung 427, Taiwan

2 School of Medicine, Tzu Chi University, Hualien 970, Taiwan

3 Graduate Institute of Chinese Medicine, School of Chinese Medicine, College of Chinese Medicine, China Medical University, Taichung 404, Taiwan

4 Department of Research, Taichung Tzu Chi Hospital, Taichung 404, Taiwan

5 Department of Nursing, Taichung Tzu Chi Hospital, Taichung 404, Taiwan
6 Office of Superintendent, Taichung Tzu Chi Hospital, Taichung 404, Taiwan

7 Department of Chinese Medicine, China Medical University Hospital, Taichung 404, Taiwan

8 Research Center for Traditional Chinese Medicine, Department of Medical Research, China Medical University Hospital, Taichung 404, Taiwan

9 Chinese Medicine Research Center, China Medical University, Taichung 404, Taiwan

10 Department of Pediatrics, Chung Kang Branch, Cheng-Ching General Hospital, No. 966, Sec. 4, Taiwan Blvd., Xitun Dist. 404, Taichung 407211, Taiwan 
visits $(P<0.001)$, days of prescription from the outpatient department $(P<0.001)$, number of emergency department visits $(P<0.001)$, number of admissions $(P<0.001)$, and days of admission $(P<0.001)$ to treat respiratory tract infections from January-September 2020 than January-September 2019. These staff members had lower numbers of outpatient department visits $(P=0.015)$ and days of prescription from the outpatient department $(P=0.009)$ to treat respiratory tract infections from January-September 2020 than January-September 2019.

Conclusion The association between our preventive measures and decreasing the risk of respiratory tract infection in nursing home residents and staff could be found. Sharing these experiences is valuable, as they provide important insights related to clinical practice during the COVID-19 pandemic.

Keywords Nursing home $\cdot$ COVID-19 $\cdot$ Taiwan $\cdot$ Preventive measures

\section{Introduction}

The coronavirus disease 2019 (COVID-19) pandemic was caused by transmission of severe acute respiratory syndrome coronavirus 2 (SARS-CoV-2), which originated in China and spread to nearby countries and then the whole world [1]. At the beginning of the COVID-19 pandemic, no one knew the proper preventive measures, including the World Health Organization (WHO). In 2003, there was an outbreak of severe acute respiratory syndrome (SARS) in Taiwan and there was not enough information to prevent and control it, too [2]. And the Taiwanese government and people learned valuable lessons from their experience with SARS about fighting emerging infectious diseases such as COVID-19. The Taiwan Central Epidemic Command Center (CECC) executed several measures to control the spread of COVID-19, such as border control, home isolation, medical mask rationing, body temperature monitoring in public areas and screening of travel history, occupation, contact history, and cluster (TOCC) before allowing people to enter medical facilities [3].

Our nursing home work together with a local hospital and their family doctors visit residents once per week. Residents and staff accept primary evaluation and treatment of the nursing home when they have medical problems. The outpatient department (OPD) visit at hospital would be allowed when they need further examination or intervention. They are transferred to the emergency department (ED) when needs of critical care or vital sign unstable. After intervention, patients and staff are discharged or admitted. COVID19 test was allowed to be administered in the ED and at admission. The staff in the nursing home could be divided into clinical (doctors, nurses and medical assistants) and nonclinical staff. All clinical staff only works in our nursing home. The doctors and nurses were trained in medical and nursing departments in the college and passed the tests of medical and nursing licenses conducted by the Taiwan Ministry of Health and Welfare (MOHW) after graduation. The medical assistants accepted training according to the guidelines from MOHW and passed the tests for practicing licenses conducted by MOHW after training programs to ensure that they had the ability to care for patients.
We summarized our preventive strategies in nursing homes during the COVID-19 pandemic (Fig. 1). Although some basic preventive measures in the day-care facility have been mentioned, but the results were hard to evaluate because of the small numbers of local cases in Taiwan. Our preventive measures are more comprehensive and we wanted to investigate the association between these measures and the difference in medical needs for respiratory infection treatment in nursing home residents and staff in 2019 and 2020 through this article.

\section{Methods}

\section{Data sources}

Our data source was electronic medical records which included all the original data, such as OPD visit, ED visits, demographic characteristics, hospitalizations, diagnosis, medical costs, interventions, and prescriptions. Medical visits to treat new respiratory tract infections were all recorded in these electronic medical records. Then, the days of prescription and admission for respiratory tract infection treatment could be identified. Diagnoses were concurrently coded according to the International Classification of Diseases, Ninth Revision, Clinical Modification (ICD-9-CM) and Tenth Revision, Clinical Modification (ICD-10-CM).

\section{Study cohort identification}

We identified residents who lived in our nursing home from Jan. 2019 to Sept. 2020 and staff who worked in the nursing home from Jan. 2019 to Sept. 2020 without interruption (Fig. 2a, b). We excluded patients or staff who had incomplete data on age or sex, and who had interrupted health insurance services during the follow-up period. Medical visits from Jan. 2019 to Sept. 2019 and Jan. 2020 to Sept. 2020 were defined as the 2019 and 2020 cohorts of residents and staff, respectively. 


\section{Before entering nursing home}

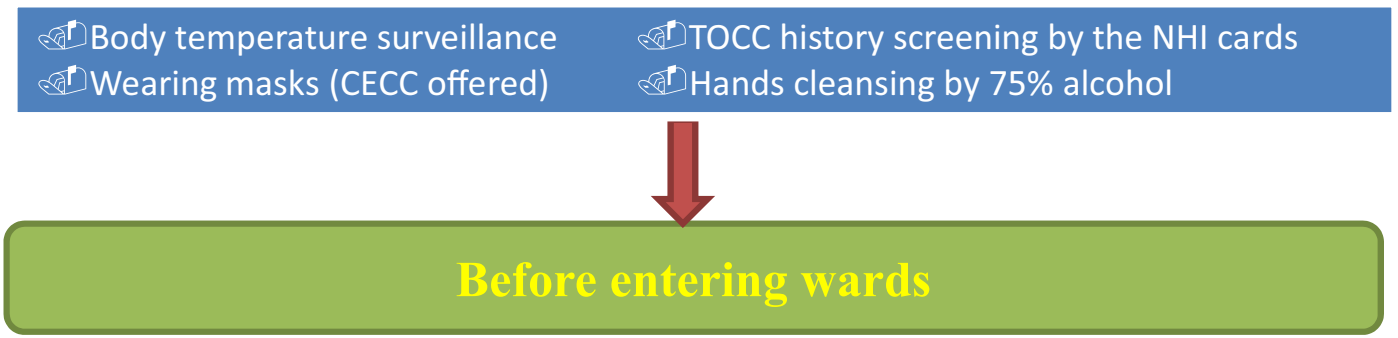

Only nursing home staff members and permitted family would be allowed to enter $75 \%$ alcohol was set around with slogan to remind users to clean their hands Cleaning the public environment (light switch and doorknobs) by $75 \%$ alcohol or disinfected water per 2 hours

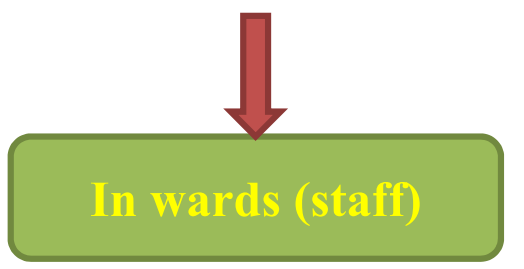

Education the latest

medical information of

COVID-19

Hands cleansing by $75 \%$

alcohol before and after

executed medical works**

Wearing medical

masks**

Wearing proper PPE (N95

masks, goggles) when

contacting with suspicious

infected environments and

residents

Keep safe distance (1.5

meter) when taking

meal/numbers of staff

members in dining table

were restricted

Office equipment

(keyboards and computer

mice) were cleaned by $75 \%$

alcohol daily

COVID-19 test would be

performed when

respiratory symptoms,

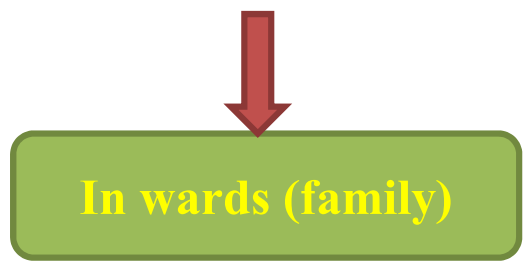

Personal contact

information were recorded

when they entering wards

Wearing masks in the

nursing home

Family with history of

traveling abroad in 14 days

would not be allowed to

enter

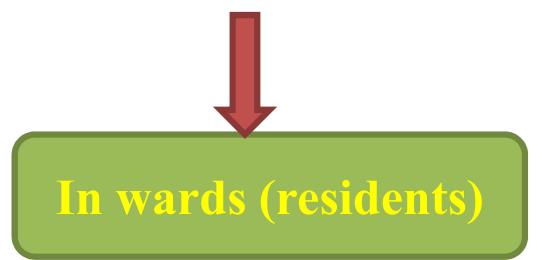

Education the

importance

of COVID19

Reminding masks

wearing

Decreasing cluster

activities

Keep safe distance when

taking meal

COVID-19 test was

performed when residents

had suspicious symptoms

Transference for medical

visits by specific vesicles

and drivers*

$75 \%$ alcohol was set in

Fig. 1 Our preventive strategies executed in the nursing home. CECC Central Epidemic Command Center, TOCC travel history, occupation, contact, and cluster, $N H I$ national health insurance, COVID-19 coronavirus disease 2019, PPE personal protective equipment 


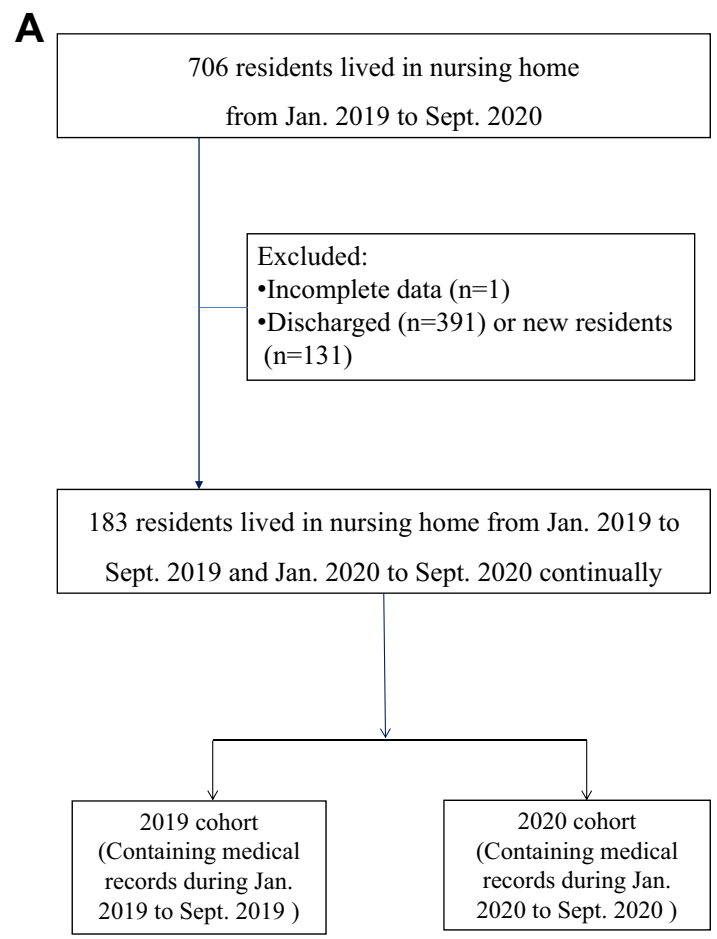

Fig. 2 a Study population flowchart. A total of 706 patients lived in our nursing home from Jan. 2019 to Sep. 2020. In the final, 183 older patients were included and their medical records were divided into two cohorts: 2019 (Jan. 2019-Sep. 2019) and 2020 (Jan. 2020-Sep. 2020) cohorts, respectively. b Study population flowchart. A total of

\section{Covariate assessment}

Sex, age, ADL scores, Charlson Comorbidity Index (CCI), duration of nursing home living, placement of nasogastric (NG) tubes, Foley catheters and need for oxygenation supplies were collected as the residents' sociodemographic factors. We divided the residents into four groups according to age ( $<60$ years, $60-69$ years, $70-79$ years, $80-89$ years, and $\geq 90$ years). The ADL scores were divided into three categories by the definition of disability (extreme disability: 0-30, moderate disability: 31-60, mild disability: 61-80). ICD-9-CM/ICD-10-CM codes of comorbidities identified more than once in the outpatient or inpatient records were taken into consideration and presented as the CCI including a weight of one (myocardial infarction, congestive heart failure, peripheral vascular disease, cerebrovascular disease, dementia, chronic obstructive pulmonary disease, connective tissue disease, peptic ulcer disease, mild liver disease, and diabetes), a weight of two (diabetes with end organ damage, hemiplegia, moderate to severe chronic kidney disease, localized solid tumor, leukemia, and lymphoma), a weight of three (moderate or severe liver disease), and a weight of six (metastatic solid tumor and acquired immune deficiency syndrome). The total score of the included residents
B

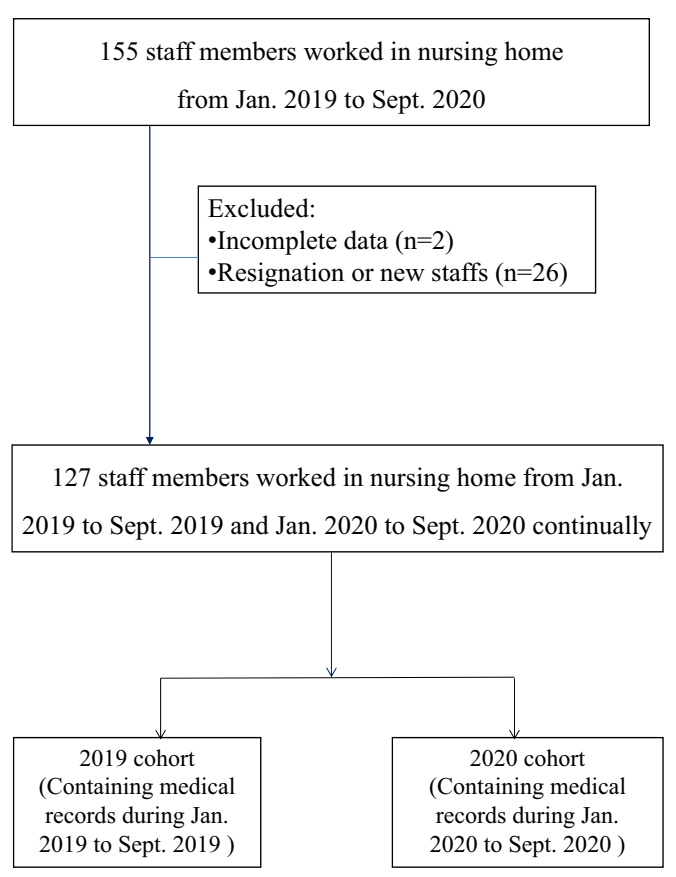

155 staff worked in our nursing home from Jan. 2019 to Sep. 2020. In the final, 127 staff members were included and their medical records were divided into two cohorts: 2019 (Jan. 2019-Sep. 2019) and 2020 (Jan. 2020-Sep. 2020) cohorts, respectively

was calculated by adding the weights and divided into three groups by CCI: $\leq 5 ; 6-9$ and $\geq 10$ [4]. The duration of nursing home living was classified into three cohorts as follows: $2-3$ years, $4-5$ years, and $\geq 6$ years.

Sex, age, and the nature of their job were the sociodemographic factors collected for the nursing home staff. They were divided into three groups by age $(<30$ years, $30-39$ years, and $\geq 40$ years). The nature of their job was defined as clinical staff or other.

\section{Definition of outcomes}

When medical records (including chief complaints, physical examinations, imaging results) of OPDs or EDs revealed that a resident (or staff) needed medical intervention because of respiratory tract infections, all OPD or ED visits were defined as "the total number of OPD visits for respiratory tract infections" and "the total number of ED visits for respiratory tract infections", respectively. All the days of prescription from OPD for respiratory tract infection treatment of each resident (or staff) were defined as "the total days with a prescription from OPD for respiratory tract infection treatment". The medical records of admission in each resident (or staff) were reviewed, and the diagnosis, imaging 
results, and treatments met the clinical presentations of respiratory tract infections before their medical visits were included in "the total number of admissions for respiratory tract infections". The days of admission in each resident (or staff) because of respiratory tract infection treatment were counted as "the total days of admission for respiratory tract infections treatment".

\section{Statistical analyses}

The baseline characteristics of the residents and nursing home staff are presented as the means (standard deviations). The 2019 and 2020 cohorts were compared using paired $t$ tests and repeated measures of ANOVA for numeric and categorical variables, respectively. The differences in subgroups of each sociodemographic factor were determined through generalized estimating equation analysis. The commercial statistical software SPSS version 22 (IBM Corp, Released 2013) was used to process the data analysis. A $P$ value $<0.05$ in a 2 -tailed test was considered statistically significant.

\section{Results}

A total of 706 residents lived in our nursing home from Jan. 2019 to Sept. 2020. We excluded residents with incomplete data $(n=1)$ or an interrupted living course $(n=522)$. The reasons for discharge are presented in the Supplementary Figure S1. In the final cohort, 183 residents were included. Table 1 shows the baseline characteristics of the included residents, including sex, age, ADL score, admission duration, CCI, need for an NG tube, Foley catheter or oxygenation supplies. Female residents $(63.9 \%)$ and residents aged $80-89$ years old were the predominant groups $(41.0 \%)$. Most participants had ADL scores $\leq 20(78.1 \%)$, and most residents lived in the nursing home for $2-3$ years (54.6\%). A CCI of $\geq 6$ was most common in these participants (95.6\%). The percentages of usage in residents were NG tubes (51.9\%) [5-7], Foley tubes (9.3\%) and oxygenation supplies (14.2\%).

The total number of OPD visits for respiratory tract infections of the included residents from Jan. 2019 to Sept. 2019 and Jan. 2020 to Sept. 2020 were 447 and 180, respectively (Supplementary Figure S2A). The mean and SD values of residents' OPD visits for respiratory tract infections from Jan. 2019 to Sept. 2019 and Jan. 2020 to Sept. 2020 were $2.44 \pm 2.97$ and $0.98 \pm 1.18$, respectively (Supplementary Figure S2B). The total days with a prescription from OPD for respiratory tract infection treatment of the included residents from Jan. 2019 to Sept. 2019 and Jan. 2020 to Sept. 2020 were 3,486 and 1,470, respectively (Supplementary Figure S3A). The mean and SD values of residents' days with a prescription from OPD for respiratory tract infection
Table 1 Characteristics of included nursing home residents

\begin{tabular}{|c|c|c|}
\hline \multirow[t]{2}{*}{ Variable } & \multicolumn{2}{|c|}{ No $(n=183)$} \\
\hline & $n$ & Percentage \\
\hline \multicolumn{3}{|l|}{ Sex } \\
\hline Male & 66 & 36.1 \\
\hline Female & 117 & 63.9 \\
\hline \multicolumn{3}{|c|}{ Age (years) } \\
\hline$<60$ & 15 & 8.2 \\
\hline $60-69$ & 20 & 10.9 \\
\hline $70-79$ & 37 & 20.2 \\
\hline $80-89$ & 75 & 41.0 \\
\hline$\geq 90$ & 36 & 19.7 \\
\hline \multicolumn{3}{|c|}{ ADL score } \\
\hline$\leq 20$ & 143 & 78.1 \\
\hline $21-60$ & 20 & 10.9 \\
\hline$\geq 61$ & 20 & 10.9 \\
\hline \multicolumn{3}{|c|}{ Admission duration (Years) } \\
\hline $2-3$ & 100 & 54.6 \\
\hline $4-5$ & 59 & 32.3 \\
\hline$\geq 6$ & 24 & 13.1 \\
\hline \multicolumn{3}{|l|}{ CCI } \\
\hline$\leq 5$ & 8 & 4.4 \\
\hline $6-9$ & 92 & 50.3 \\
\hline$\geq 10$ & 83 & 45.4 \\
\hline \multicolumn{3}{|c|}{ Nasogastric tubes placement } \\
\hline Yes & 95 & 51.9 \\
\hline No & 88 & 48.1 \\
\hline \multicolumn{3}{|c|}{ Foley tubes placement } \\
\hline Yes & 17 & 9.3 \\
\hline No & 166 & 90.7 \\
\hline \multicolumn{3}{|c|}{ Oxygen supply } \\
\hline Yes & 26 & 14.2 \\
\hline No & 157 & 85.8 \\
\hline
\end{tabular}

No number, $A D L$ activities of daily living, $C C I$ Charlson Comorbidity Index

treatment from Jan. 2019 to Sept. 2019 and Jan. 2020 to Sept. 2020 were $19.05 \pm 26.19$ and $8.03 \pm 17.16$, respectively (Supplementary Figure S3B). The total number of ED visits for respiratory tract infections of the included residents from Jan. 2019 to Sept. 2019 and Jan. 2020 to Sept. 2020 were 93 and 42, respectively (Supplementary Figure S4A). The mean and SD values of the residents' ED visits for respiratory tract infections from Jan. 2019 to Sept. 2019 and Jan. 2020 to Sept. 2020 were $0.51 \pm 0.89$ and $0.23 \pm 0.56$, respectively (Supplementary Figure S4B). The total number of admissions for respiratory tract infections of the included residents from Jan. 2019 to Sept. 2019 and Jan. 2020 to Sept. 2020 were 79 and 33, respectively (Supplementary Figure S5A). The mean and SD values of the number of admissions for respiratory tract infections of the included residents from 
Jan. 2019 to Sept. 2019 and Jan. 2020 to Sept. 2020 were $0.43 \pm 0.85$ and $0.18 \pm 0.45$, respectively (Supplementary Figure S5B). The total days of admission for respiratory tract infections treatment of the included residents from Jan. 2019 to Sept. 2019 and Jan. 2020 to Sept. 2020 were 1,009 and 387, respectively (Supplementary Figure S6A). The mean and SD values of the residents' days of admission for respiratory tract infection treatment from Jan. 2019 to Sept. 2019 and Jan. 2020 to Sept. 2020 were $5.51 \pm 11.88$ and $2.11 \pm 5.60$, respectively (Supplementary Figure S6B).

Supplementary Tables S1, S2, S3, S4, and S5 show the results of a paired sample $t$ test comparing the number of OPD visits, the days of a prescription from the OPD, the number of ED visits, the number of admissions, and the days of admission for respiratory tract infection treatment from Jan. 2019 to Sept. 2019 and Jan. 2020 to Sept. 2020, respectively. When compared with Jan. 2019 to Sept. 2019, the number of OPD visits, the days of a prescription from the OPD, the number of ED visits, the number of admissions, and the days of admission were lower from Jan. 2020 to Sept. $2020(P<0.001)$.

A total of 155 staff members continuously worked in our nursing home from Jan. 2019 to Sept. 2020. We excluded staff with incomplete data $(n=2)$ or interrupted services $(n=26)$ from Jan. 2019 to Sept. 2020. In the final cohort, 127 staff members were included. Table 2 shows the baseline characteristics of the included staff, including sex, age, and the nature of their job. Female staff (85\%) and staff aged $<30$ years old were the predominant groups (41.7\%). Most of the participants were clinical staff $(92.1 \%)$.

The total OPD visits for respiratory tract infections of the included staff from Jan. 2019 to Sept. 2019 and Jan. 2020 to Sept. 2020 were 50 and 23, respectively (Supplementary Figure S7A). The mean and SD values of the number of OPD visits for respiratory tract infections of the staff from Jan. 2019 to Sept. 2019 and Jan. 2020 to Sept. 2020 were

Table 2 Characteristics of nursing home staff members

\begin{tabular}{lcl}
\hline Variable & No $(n=127)$ & \\
\cline { 2 - 3 } & $n$ & Percentage \\
\hline Sex & 19 & 15 \\
$\quad$ Male & 108 & 85 \\
Female & & \\
Age (years) & 53 & 41.7 \\
$\quad<30$ & 31 & 24.4 \\
$30-39$ & 43 & 33.9 \\
$\geq 40$ & & \\
Clinical staffs & 117 & 92.1 \\
$\quad$ Yes & 10 & 7.9 \\
$\quad$ No & & \\
\hline
\end{tabular}

No number
$0.39 \pm 1.00$ and $0.18 \pm 0.56$, respectively (Supplementary Figure S7B). The total days with a prescription from OPD for respiratory tract infection treatment of the included staff from Jan. 2019 to Sept. 2019 and Jan. 2020 to Sept. 2020 were 370 and 133, respectively (Supplementary Figure S8A). The mean and SD values of the days with a prescription from OPD for respiratory tract infection treatment of the staff from Jan. 2019 to Sept. 2019 and Jan. 2020 to Sept. 2020 were $2.91 \pm 7.92$ and $1.05 \pm 3.22$, respectively (Supplementary Figure S8B).

Supplementary Tables S6 and S7 show the results of a paired sample $t$ test comparing the number of OPD visits and the days of a prescription from the OPD for respiratory tract infection treatment from Jan. 2019 to Sept. 2019 and Jan. 2020 to Sept. 2020, respectively. When compared with Jan. 2019 to Sept. 2019 , the number of OPD visits $(P=0.015)$, and the days of a prescription from the OPD $(P=0.009)$ were lower from Jan. 2020 to Sept. 2020.

Decreasing trends of the number of OPD visits, the days of a prescription from the OPD, the number of ED visits, the number of admissions, and the days of admission for respiratory tract infection treatment in the included residents from Jan. 2020 to Sept. 2020 stratified by month are presented in Supplementary Figures S9A, S9B, S10, S11A, and S11B.

Supplementary Table $\mathrm{S} 8$ shows the research results using generalized estimating equation (GEE) analysis to examine the relationship between various factors, including sex, age, ADL score, admission duration, CCI, need for an NG tubes, Foley catheters or oxygenation supplies in the residents. Compared to the male cohort, the female cohort had a significant negative correlation with the total number of ED visits [95\% confidence interval $(\mathrm{CI}):-1.47$ to -0.55 , $P<0.001$ ] or the total days of admission (95\% CI: -1.35 to $-2.34, P=0.006)$ for respiratory tract infections. Compared to residents who were $<60$ years, $60-69$ years had a significant negative correlation with the total number of OPD [95\% confidence interval $(\mathrm{CI}):-2.45$ to $-0.08, P<0.001$ ] or the total days with a prescriptions from OPD $(95 \% \mathrm{CI}$ : -9.97 to $-2.07, P=0.003$ ) for respiratory tract infections. Compared to residents with ADL scores $\leq 20$, those who with ADL scores $\geq 61$ had a significant negative correlation with the total number of admissions (95\% CI -2.95 to $-1.11, P<0.001)$ for respiratory tract infections. Compared to residents with an admission duration of 2-3 years, those with $\geq 6$ years had a significant negative correlation with the total number of ED visits ( $95 \%$ CI -3.64 to -0.58 , $P=0.007)$, the total number of admissions (95\% CI -1.84 to $-1.41, P=0.002)$ or the total days of admission $(95 \% \mathrm{CI}$ -3.75 to $-0.82, P=0.002$ ) for respiratory tract infections. When we analyzed these 5 outcomes of residents through GEE, there was no group difference in CCI or the need for a Foley tube. Compared to residents with NG tubes, those who did not have NG tube had significant positive correlation 
with the total number of OPD ( $95 \%$ CI $0.32-1.43, P=0.002$ ) and the total days with a prescriptions from OPD (95\% CI $0.68-3.22, P=0.003$ ); and a significant negative correlation with the total number of ED visits $(95 \% \mathrm{CI}-2.07$ to -0.68 , $P<0.001$ ), or the total days of admission(95\% CI -2.39 to $-0.92, P<0.001)$ for respiratory tract infections. Compared to residents who needed oxygenation supply, those without the needs had a significant negative correlation with the total days with a prescription from OPD $(95 \% \mathrm{CI}-4.31$ to $-0.32, P=0.023$ ) for respiratory tract infections.

The research results using GEE analysis to examine the relationship between various factors, including sex, age, and the nature of their job in the staff, are presented in Supplementary Table S9. When we analyzed the 2 outcomes of staff through GEE, there was no group difference in sex. Compared to staff who were $<30$ years, $30-39$ years (95\% CI -3.37 to $-0.48, P=0.009)$ and $\geq 40$ years $(95 \% \mathrm{CI}-2.27$ to $-0.27, P=0.013$ ) had significant negative correlations with the total days with a prescription from OPD for respiratory tract infections, respectively. Compared to clinical staff, nonclinical staff had a significant positive correlation with the total number of OPD ( $95 \%$ CI $0.83-7.30, P=0.014)$ and the total days with a prescription from OPD (95\% CI $0.71-2.95, P=0.001)$ for respiratory tract infections.

\section{Discussion}

COVID-19 outbreaks have occurred in nursing homes worldwide [8] and preventive measures to protect nursing home residents from COVID-19 are becoming important. We attempted to design our strategies based on the experience of fighting SARS and suggestions from the Taiwanese CECC. Our strategies were not only for the residents but also for the staff and families. TOCC screening using personal NHI cards is a unique Taiwanese experience, and is useful to detect a high risk population before they enter medical facilities [3]. Because most Taiwanese people still remember their experience with the SARS outbreak in Taiwan, they easily accepted the policies enforcing body temperature monitoring, wearing masks and cleaning hands with $75 \%$ alcohol even though the evidence for decreasing the spread of COVID-19 by wearing masks had not been determined at that time. In Jan. 2020, the first confirmed COVID-19 case was diagnosed in Taiwan, and we limited the number of visitors for the nursing home residents. As the number of confirmed COVID-19 cases rose in Taiwan, no unnecessary visitors were allowed to visit the nursing home. People who were permitted to enter the nursing home were asked to leave their name, cell phone number and address for tracing. However, visitation applications were not allowed later because the spread of the COVID-19 pandemic in nursing homes in European countries showed troublesome results
[9]. The medical services in our nursing home used to be offered by fixed healthcare providers, which is helpful in preventing cross-infection during the COVID-19 pandemic. The family doctors came to our nursing home to evaluate the residents, and the residents' medicine was transported by specific staff, which decreased unnecessary hospital visits. COVID-19 tests were suggested to residents when they were readmitted to the nursing home after discharge from the hospital, especially those who had a contact history with a suspicious case. Through these steps, we were able to confirm that all of our cases had the least risk of being infected.

It was essential to introduce COVID-19 information to the residents and staff before we asked them to follow these preventive measures. Although wearing masks and cleaning hands with $75 \%$ alcohol were already routine practices for the nursing home staff, we still regularly invited staff to check their ability to wear PPE during the regular meetings. PPE including medical masks, was supplied by the Taiwan CECC and offered to each nursing home staff and resident. When they removed their masks, they were required to keep a safe distance $(1.5 \mathrm{~m})$ between each other in spaces such as the dining area. We encouraged our staff to clean their hands with $75 \%$ alcohol not only when they were providing medical care but also when they were in contact with the public environment, such as touching light switch and doorknobs. To promote hand cleaning, many new $75 \%$ alcohol machines were placed in public areas. When residents entered the living room on each floor, they were asked to wear a medical mask. Those who had respiratory symptoms were asked to wear their masks in their bedroom. The Taiwan CECC also asked healthcare providers to take a COVID-19 test in the ED when they had a fever, respiratory tract symptoms, diarrhea, or other suspicious symptoms. This might possibly explain why there was no difference in ED visits between 2019 and 2020 (in 2020 there were six ED visits and five of them were preventive COVID-19 tests). Cleaning the environment is also another important preventative measure; we have found that the coronavirus can live in the environment for hours after it leaves the human body [10]. We followed the suggestion from the Taiwan CECC and used 1,000 ppm bleach $[100 \mathrm{ml}$ bleach (brand name: Shanyi, concentration of $\mathrm{NaOCl}: 12 \%$ ) in $12 \mathrm{~L}$ tap water] to clean the environment of the nursing home every $2 \mathrm{~h}$.

Evaluating the result after these preventive measures were performed is important. Because the COVID-19 outbreak in Taiwan was restricted, we used the trends of medical needs of respiratory tract infections in the residents and nursing home staff to observe the effectiveness of our measures. There was no difference in organization, staff resources or casual care between 2019 and 2020 in our nursing home. We only executed COVID-19 preventive measures in 2020, which could indicate that the differences in our outcomes could come from these measures. When residents needed 
further treatment for respiratory tract infections, they were arranged for admission and the criteria were the same in 2019 and 2020. Isolation rooms were prepared for suspected COVID-19 cases during admission. The ED is the primary unit for further admission. We seldom allow patients to admit through OPD, expect for some planned treatments, such as chemotherapy. The trends of medical needs of visits to the ED and admission, stratified by month were more compatible than the trends of times of visits to the OPD and admission, stratified by month. The number of OPD visits, the days of a prescription from the OPD, the number of ED visits, the number of admissions, and the days of admission for respiratory tract infection treatments in residents were significantly lower in $2020(P<0.001)$. The results were independent of the residents' sex, age, ADL score, CCI, admission duration, Foley tubes and oxygenation supply. The number of OPD visits $(P=0.015)$, and the days of a prescription from the OPD $(P=0.015)$ for respiratory tract infection treatment in nursing home staff were also significantly lower in 2020. Few individuals visited the ED for respiratory tract infections and the policy of asking nursing home staff to be tested for COVID-19 in the ED in 2020 may have caused no statistical significance in the numbers of ED visits between 2019 and 2020 .

The multivariate analyses were presented through GEE analysis which performed in the residents and staff cohorts. Compared to the male residents, female residents had few times to accept admission therapy for respiratory tract infection. The difference of age subgroup could only be found in outcomes of "the total number of OPDs" and "the total days with a prescription from OPDs" for respiratory tract infection. Compared to residents living in nursing homes $2-3$ years, those who lived $\geq 6$ years with few medical needs for ED visits or admissions for respiratory tract infection. Compared to residents with NG tubes, those without NG tubes had more medical visits for OPDs, but fewer medical needs in EDs or admissions for respiratory tract infection treatment. Compared to staff aged $<30$ years old, those aged $\geq 30$ years old had fewer prescription days from OPD for respiratory tract infection treatment. Compared to clinical staff, nonclinical staff had more medical needs to visit OPDs for respiratory tract infection treatment. These findings may reflect that older staff executes preventive measures more comprehensively than younger staff. Similar presentations of the association of compliance with executing preventive measures to fight COVID-19 and differences in age have been reported from the data of 27 countries [11].Clinical staff could identify the disease presentation in the residents and they could use adequate PPE to protect them from infection.

There were several limitations to our study. The number of participants was small; however, it had detailed medical visit records of the residents and staff. The follow-up period was not long enough, and we will continue to follow-up the course of respiratory tract infections in these participants throughout the entire COVID-19 pandemic. Residents may have used drugs for respiratory tract infection treatment offered by their families; however, the numbers of visitors were limited, and we always recorded these medicines in the medical records and did not find such a situation to date. The causal relation between our preventive measures and outcomes could not be proven directly from a retrospective study design. However, their relationship could still be noted after our analysis.

In European, limited or part-time numbers of staff are obstacles to execute preventive measures in the nursing homes [12]. A shortage of PPE has been found to be one of the problems in the United States in the COVID-19 pandemic [13]. The difficulty of maintaining proper distance has been found in nursing homes in Korea [14]. Our preventive strategies for nursing homes included well-organization, surveillance of vital signs and TOCC screening at entrance, specific staff for caring, timely education, proper PPE wearing, maintaining social distance, and environmental disinfection comprehensively. We administered a COVID-19 test to suspected residents and staff. After we began our strategies in Jan. 2020, we observed fewer OPD or ED medical visits, days of prescriptions from OPD, admission times and lengths for treating respiratory tract infections in our residents one month later. The nursing home staff could have also benefited from these strategies because they had fewer OPD medical visits and shorter days of prescription for respiratory tract infection. Although our residents have not been diagnosed with COVID-19, it is still notable to share our experience because of their effectiveness in decreasing the risk and severity of respiratory tract infections in nursing home residents and staff.

Supplementary Information The online version contains supplementary material available at https://doi.org/10.1007/s41999-021-00459-7.

Author contributions $\mathrm{CH}$ designed, performed the research and helped writing the manuscript. $\mathrm{CH}$ and YK analyzed the manuscript. SC supervised the study. HY supervised the study and finalized the manuscript. ST conceptualized the study, drafted the manuscript and finalized the manuscript.

Funding Not applicable.

\section{Compliance with ethical standards}

Conflict of interest All the authors do not have any conflict of interest.

Ethical approval Our research did not belong to human subject research or animals study.

Informed consent Waiver of informed consent was applied because of the study design was medical records review, personal information was not disclosed, and included patients had lowest risk. 


\section{References}

1. Ge H, Wang X, Yuan X, Xiao G, Wang C, Deng T et al (2020) The epidemiology and clinical information about COVID19. Eur J Clin Microbiol Infect Dis. https://doi.org/10.1007/ s10096-020-03874-Z

2. Yen MY, Chiu AH, Schwartz J, King CC, Lin YE, Chang SC et al (2014) From SARS in 2003 to H1N1 in 2009: lessons learned from Taiwan in preparation for the next pandemic. J Hosp Infect 87(4):185-193. https://doi.org/10.1016/j.jhin.2014.05.005

3. Wang CJ, Ng CY, Brook RH (2020) Response to COVID-19 in Taiwan: big data analytics, new technology, and proactive testing. JAMA 323(14):1341-1342. https://doi.org/10.1001/jama.2020. 3151

4. Buntinx F, Niclaes L, Suetens C, Jans B, Mertens R, Van den Akker M (2002) Evaluation of Charlson's comorbidity index in elderly living in nursing homes. J Clin Epidemiol 55(11):1144 1147. https://doi.org/10.1016/s0895-4356(02)00485-7

5. Chang WK, Lin KT, Tsai CL, Chung CH, Chien WC, Lin CS (2016) Trends regarding percutaneous endoscopic gastrostomy: a nationwide population-based study from 1997 to 2010 . Medicine 95(24):e3910. https://doi.org/10.1097/MD.0000000000003910

6. Yeh L, Lo LH, Fetzer S, Chen CH (2010) Limited PEG tube use: the experience of long-term care directions. J Clin Nurs 19(19-20):2897-2906. https://doi.org/10.1111/j.1365-2702.2009. 03157.x

7. Lin LC, Wu SC, Chen HS, Wang TG, Chen MY (2002) Prevalence of impaired swallowing in institutionalized older people in taiwan. J Am Geriatr Soc 50(6):1118-1123. https://doi.org/10. 1046/j.1532-5415.2002.50270.x

8. Li Y, Temkin-Greener H, Shan G, Cai X (2020) COVID-19 infections and deaths among Connecticut nursing home residents: facility correlates. J Am Geriatr Soc 68(9):1899-1906. https:// doi.org/10.1111/jgs.16689

9. O'Neill D, Briggs R, Holmerová I, Samuelsson O, Gordon AL, Martin FC (2020) COVID-19 highlights the need for universal adoption of standards of medical care for physicians in nursing homes in Europe. Eur Geriatr Med 11(4):645-650. https://doi.org/ 10.1007/s41999-020-00347-6

10. Silverman AI, Boehm AB (2020) Systematic review and metaanalysis of the persistence and disinfection of human coronaviruses and their viral surrogates in water and wastewater. Environ Sci Technol Lett 7(8):544-553. https://doi.org/10.1021/acs.estle tt.0c00313

11. Daoust JF (2020) Elderly people and responses to COVID-19 in 27 Countries. PLoS ONE 15(7):e0235590. https://doi.org/10. 1371/journal.pone. 0235590

12. Grabowski DC, Mor V (2020) Nursing home care in crisis in the wake of COVID-19. JAMA 324(1):23-24. https://doi.org/10. 1001/jama.2020.8524

13. Szczerbińska K (2020) Could we have done better with COVID19 in nursing homes? Eur Geriatr Med 11(4):639-643. https://doi org/10.1007/s41999-020-00362-7

14. Song R, Kim HS, Yoo SJ, Lee K, Park JH, Jang JH et al (2020) COVID-19 in nursing facilities: experience in Republic of Korea. Osong Public Health Res Perspect 11(4):164-169. https://doi.org/ 10.24171/j.phrp.2020.11.4.04

Publisher's Note Springer Nature remains neutral with regard to jurisdictional claims in published maps and institutional affiliations. 